\title{
Human-like Reflexes for Robotic Manipulation using Leaky Integrate-and-Fire Neurons
}

\author{
Christian Bauer, Giulio Milighetti, Wenjie Yan, Ralf Mikut
}

\begin{abstract}
In this paper we present an approach to transfer human-like reflex behavior to robots by utilizing leaky integrate-and-fire neurons. For the acceptance of robots in general and humanoid robots, which are even closer to people's daily life, in particular a main aspect is their appearance and how they act and move in human centered environments. Especially safety strategies are crucial for a widespread acceptance of these machines. In our work we target this safety aspect by approaching this issue from the direction how humans respond to external stimuli. To achieve such human-like reflexes a general reflex unit, based on special variants of the leaky integrate-and-fire neuron model has been built. Instances of this reflex unit are adapted to special reflex types and connected to form dependent reflex behaviors. The concept of these neural structures and its evaluation by means of several experiments are presented in this paper. The results are depicted in detail and future aspects of our ongoing work are addressed.
\end{abstract}

\section{INTRODUCTION AND MOTIVATION}

Like many other research projects about humanoid robotics the collaborative research project "Humanoid Robots" (SFB-588) of the German Research Foundation (DFG: Deutsche Forschungsgemeinschaft) has the goal to enable anthropologically designed robots to work in human centered environments. One key issue for a better acceptance of humanoid robots by the users and human environment is the safety aspect. A robot has to act without harming anyone or damaging objects. For this task different strategies can be applied and they range from motion capturing based techniques to programming by presentation.

In this paper a different approach to the afore mentioned is presented, targeting the subject of human like movements and safety strategies from the neurobiological point of view. A human-like reflex behavior has been simulated by leaky integrate-and-fire neurons, a special variant of an artificial neuron model, and was tested successfully.

Other works in this area are the research of Folgheraiter [1] who applied a neural control to an anthropomorphic hand to control the stiffness and to emulate myotatic human reflexes.

This work was supported by Deutsche Forschungsgemeinschaft (DFG) Collaborative Research Project 588

Chr. Bauer is member of the Institute of Applied Computer Science at the Karlsruhe Institute of Technology, Karlsruhe, Germany christian.bauerakit.edu

Giulio Milighetti is member of the Fraunhofer Institute of Optronics, System Technologies and Image Exploitation IOSB, Karlsruhe, Germany giulio.milighetti@iosb. fraunhofer.de

W. Yan was member of the Institute of Applied Computer Science at the Karlsruhe Institute of Technology, Karlsruhe, Germany alex1984.yan@gmail. com

R. Mikut is member of the Institute of Applied Computer Science at the Karlsruhe Institute of Technology, Karlsruhe, Germany ralf.mikutakit.edu
Yigit [2] used a more technical approach to applying low level behaviors to a robot arm, in order to achieve motions comparable to human reflexes. Kawasaki and Mouri [3] developed specialized reflexes for their anthropomorphic hand based on force and velocity control, and Shimoda [4] used biological control features to realize bipedal walking without trajectory planning. In our work, we designed a special type of artificial neural circuit which is capable to function as a reflex on a humanoid robot, especially the demonstrator platform ARMAR III (Fig. 1) [5]. The chosen approach is a

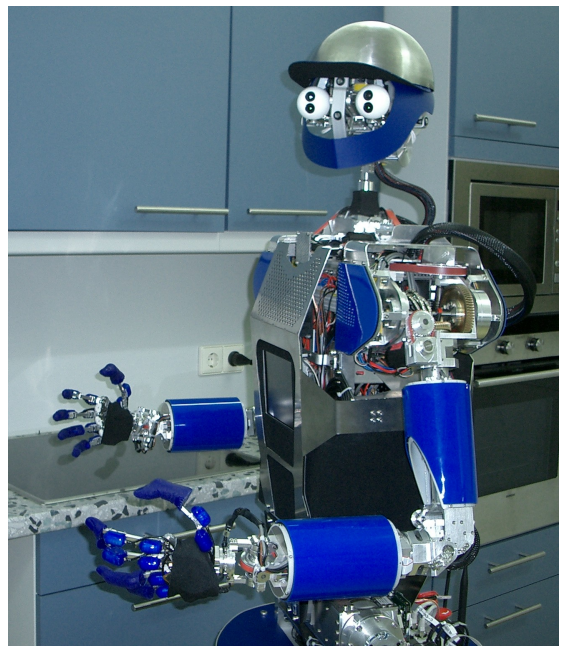

Fig. 1. Robot ARMAR IIIa of the SFB-588

very general one, which enables the specialization of the reflexes to different tasks and makes it possible to build complex reflex networks in which the reflexes depend on each other.

This work is part of our research to build a cognition architecture for humanoid robots [6], based on a Petri-net based supervisory control concept $[7,8]$.

In Section II the leaky integrate-and-fire neuron model is introduced, as well as two specialized variants of this model which best suit our purposes. Section III introduces how these reflexes are built, based on the more general prototype, and several examples are shown. In addition to that a dependency network consisting of three reflexes is shown. Section IV describes the hardware of the demonstrator platform at the Fraunhofer IOSB and specifies the performed experiments. The evaluation and the achieved results are then presented in Section V followed by Section VI in which this paper is concluded and a lookout to our future work is given. 


\section{METHODS}

Several different neural models exist that are suitable for the task of modeling the behavior of biological neurons. An overview of different models is given in [9] which ranges from Hodgkin-Huxley models $(\mathrm{HH})$ to leaky integrate-andfire models (LIF). The HH model [10] uses several coupled differential equations to simulate biological neurons very realistic, but this rises the computation time and restricts the possibilities for applications. The FitzHugh-Nagumo model (FN) [11] is a simplified variant of $\mathrm{HH}$ model but regarding the task we are looking for it is still too complex. Another simplification of the $\mathrm{HH}$ model is the leaky integrate-andfire model (LIF) [12] which relies on the variability of the current. This model is just event triggered and different specializations exist. The Spike-Response model (SRM) [13, 14] is a generalization of the LIF model and integrates the activation as well as the refractability into its computations. For the task of building reflex behavior the LIF model fits the requirements best, such as it only relies on the variability of the current and its event triggered behavior [15].

The basic structure of the integrate-and-fire neuron can be described as a circuit consisting of a capacity $C$ in parallel with a resistor $R$ [14]. These are driven by current $I(t)$ as external input of the neuron $i$ with $\tau_{m}=R C$ as a time constant "Leakage Integrator". For nonlinear models follows:

$$
\tau_{m} \frac{d u_{i}}{d t}=F(u)+R_{G}(u) I_{i}(t) \text { for all } i
$$

In this $R_{G}(u)$ represents the current dependent resistor and $F(u)$ is a nonlinear function to describe the membrane potential. The components $R_{G}(u)$ and $F(u)$ can be defined as time dependent functions. If a neuron can be exited not only by external influences but also by neighboring neurons, the synaptic connections have to be simulated as well. The mathematic representation of these synaptic currents is given by $I_{i}(t)$ and described as follows with $v(t)$ as threshold:

$$
I_{i}(t)=\sum_{j} w_{i j} \sum_{f} \alpha\left(t-t_{j}^{(f)}\right) \text { with } t_{j}^{(f)}: u\left(t_{j}^{(f)}\right) \geq v\left(t_{j}^{(f)}\right)
$$

$w_{i j}$ is used as weighting factor for the effectiveness of the synaptic connections. At time $t_{j}^{(f)}$ the activation of neuron $j$ takes place, saying that, if the presynaptic neuron $j$ fires, the postsynaptic neuron $i$ registers a current, too. The value of this current $\alpha\left(t-t_{j}^{(f)}\right)$ can be calculated by:

$$
\alpha\left(t-t_{j}^{(f)}\right)=-g\left(t-t_{j}^{(f)}\right)\left(u_{i}(t)-E_{s y n, i j}\right)
$$

where $g\left(t-t_{j}^{(f)}\right)$ is the conductance, which is changing over time.

The reverse potential $E_{s y n, i j}$ depends on the synaptic type in use. For excitatory synapses $E_{s y n, i j}$ is much higher than the reset potential $u_{r}$. For $u_{i}(t)<E_{s y n, i j}, \alpha$ will be positive, which induces a positive presynaptic voltage and the membrane potential rises. The higher the voltage, the lower the amplitude of the input. For inhibitory synapses the reverse potential lies closer to the reset potential. If an action potential arrives at an inhibitory synapse the membrane potential is driven in the direction of the reverse potential. Ergo, if a neuron is reset the inhibitory input has only little influence on the membrane potential. In contrary, if the membrane potential is much higher than $u_{r}$, a strong inhibitory effect is applied.

A LIF model is described implicitly by the form of its action potentials. Spikes are now called "fire-event" and the threshold represents the time at which such a fire-event takes place:

$$
t_{i}^{(f)}: \quad u\left(t_{i}^{(f)}\right)=\vartheta \text { with } v(t)=\text { const }=\vartheta
$$

The membrane potential will fall to the new value $u_{r}<\vartheta$ after time point $t^{(f)}$. The refractability describes the ability of these artificial neurons to lay silent after an activation for a certain time $t_{\text {silent }}$. In this time, the neuron can not be activated and the input $I(t)$ is suppressed, the membrane potential without any input converges to zero. Shortly after an activation the potential falls to the reset potential and then converges to the zero point, while input signals arrive constantly. The suppression is mathematically represented by:

$$
\tau_{m} \frac{d u}{d t}=-u(t)
$$

With this, the membrane potential for a regular LIF neuron can be described completely as:

$$
u_{i}= \begin{cases}\vartheta & \text { for } t=t_{i}^{(f)} \\ u_{\text {spike }} & \text { for } t=t_{i}^{(f)}+\varepsilon, \varepsilon \rightarrow 0 \\ (5) & \text { for } t \in\left[t_{i}^{(f)}, t_{i}^{(f)}+t_{\text {silent }}\right] \\ (1) & \text { else }\end{cases}
$$

$u_{\text {spike }}$ is the spike potential, to which the membrane potential jumps if the neuron fires. The ability to adapt dynamically is a core attribute of neurons. The intervals between activation and transmission of information depend on the correlation between spikes [16].

In our work, two different specializations of the LIF model are used to build a general reflex unit which then can be specialized to specific, different reflexes. The first specialization is the LIF model with dynamical threshold [16, 17] and the second is the LIF model with adaptive current [18] which gives an alternative to the first one, but was not used do build the reflexes introduced here. Simplifications have been integrated to increase the performance of the computations [15].

The LIF model with dynamical threshold takes into account that the membrane potential as well as the threshold change over time. The changing of the threshold is coupled with the spike signals to represent the dependency regarding the activation frequency. One simple model for this is that in addition to the membrane potential, like in (1), the threshold is adapted during activation:

$$
\tau_{v} \frac{d v_{i}}{d t}=-\left(v_{i}(t)-v_{0}\right)+u_{f}(t)
$$

This changes the calculation of the membrane potential $u_{i}$ when the neuron reaches the threshold $v(t)$ at $t=t_{i}^{(f)}$, with 
$\delta(t)$ Dirac delta impulse:

$$
v(t)= \begin{cases}(7) \text { with } u_{f}=u_{\text {spike }} \cdot \delta(t) & t=t_{i}^{(f)} \\ (7) \text { with } u_{f}=0 & \text { else }\end{cases}
$$

In Fig. 4 the potential signal of the LIF neuron with dynamical threshold of the tactile sensor is shown. The more it is activated after seconds 9 and 13 , the more the threshold increases which aggravates the reaching of it. The activation signal is triggered each time the threshold is reached. If the threshold is updated with the input signal $I(t)$ and not with the activation signal $u_{f}(t)$ the calculation of the membrane potential $u_{i}(t)$ during the refractory phase is done according to (1) and the threshold $v(t)$ is computed as shown in (7) with $u_{f}=R_{G}(u) I(t)$.

If the threshold $v(t)$ is connected to the input signal $I(t) . \tau_{m}$ and $\tau_{v}$ describe the conductances of the membrane potential and the threshold. If the input signal $I(t)$ has a constant value $I$ the membrane potential $u(t)$ converges to $I$ and the threshold $v(t)$ to $I+v_{0}>I$, because the zero point of the threshold is greater than zero. With this ability a neuron can suppress the activation after a limit cycle with constant input. One problem of this modification is, that the membrane potential can start to oscillate if the input signal is big. In this case the suppression fails, because of the dependency between activation potential and reset potential. This makes the application of a signal buffer necessary which stores the signals on activation for replacing the reset potential.

\section{THE REFLEX SYSTEM}

To apply this general form of reflex to a real robot, specific reflexes need to be defined. For a humanoid robot, such as ARMAR III, the capability of handling different objects is a key functionality. Grasping and slipping reflexes are two of the main reflexes involved here. To guarantee safety in the human-robot-interaction, also a collision reflex has been implemented but it will not be described in further detail in this paper. After that, the built reflex network and its dependencies are explained. To realize these reflexes special sensors are necessary to detect if an object is placed into the fingers respectively gripper and if slipping occurs. In addition, forces and torques in the wrist have to be recorded to detect collisions and to adapt the motor control if an object is manipulated.

\section{A. Specific reflexes}

Grasp reflex: The grasp reflex focuses on the execution of automatic grasping actions which even infants are capable of. If a finger is placed onto the palm of an infant it closes the hand autonomously. This human reflex reaction has a latency of approximately $0.5-1.5 \mathrm{sec}$. [19]. This is why this reflex should be not too sensitive.

Slip reflex: Once an object is grasped the slip reflex comes into play. This reflex detects the slipping of a grasped object and tightens the grip to prevent the object from slipping out of the grip. This reflex completely depends on a successfully performed grasp of an object. Otherwise it is deactivated.
Collision reflex: This reflex takes care of handling unforeseen collisions of the robot arm with its environment triggering a retraction of the robot arm.

All reflexes have in common, that the values of the according sensors are merged, and, if applicable, stored to a buffer. After that they are transmitted to the neuron model of the reflex which triggers the reflex based on the calculation of the membrane potential and if it reaches the threshold. As an example, the slip reflex records the positioning signals of a grasped object and compares them to the previous position. The focus of attention lies on the changing of these values. Additional considerations regarding the other two reflexes can be found in [20].

\section{B. Reflex system}

Taking into account the existing dependencies between the introduced reflexes a coordination framework has to be designed. In general a reflex model depends on the incoming sensor signals and the decision, if a reflex is triggered or not is based on the values of these signals. This decision can be handled by single neuron models, e.g. the triggering of a collision reflex, but also more complex reflex systems can be built by combining several neuron models. An example for this is grasping and holding an object. Depending on the relevancy of different reflexes, priorities are assigned and coordination is necessary to prevent the system from conflicts which might occur during activation of different reflexes at the same time.

The dependencies of the three reflexes are described below. The structure of the reflex system is shown in Fig. 2. Sensor signals are filtered and stored to a buffer. Then they are forwarded to the LIF neurons. If the threshold is reached, the neuron fires and the according signal is triggered. This leads to the decision process "Make Decision" where the reflex model decides, based on the actual environment if the reflex signal is processed further. The decision functions depend on the according reflex models. The reflex is triggered as soon as its conditions are met. These conditions are explained in the following.

\section{Decision functions}

Slip reflex: The slip reflex is only allowed to be activated after the grasp reflex has finished its task successfully (state "grasp end" is active). If the reflex model of the slipping sensor is activated again after finishing the grasping task, the activation signal is forwarded to the slip reflex and the gripper is closed tighter.

Grasp reflex: Because of its complexity the grasp reflex uses two different sensors. One is a tactile sensor, the other a sensor which is capable of detecting movements of grasped objects. Two state buffers are used to detect the actual grasping situation. Grasping is started if one of the relevant sensors is activated. Depending on which sensor is activated, the state "grasp reflex (S)" (for the slip sensor) or "grasp reflex (T)" (for the tactile sensor) is set to true and the gripper is closed continuously. As soon as the second sensor sends its firing signal, the closing of the gripper is stopped and the 


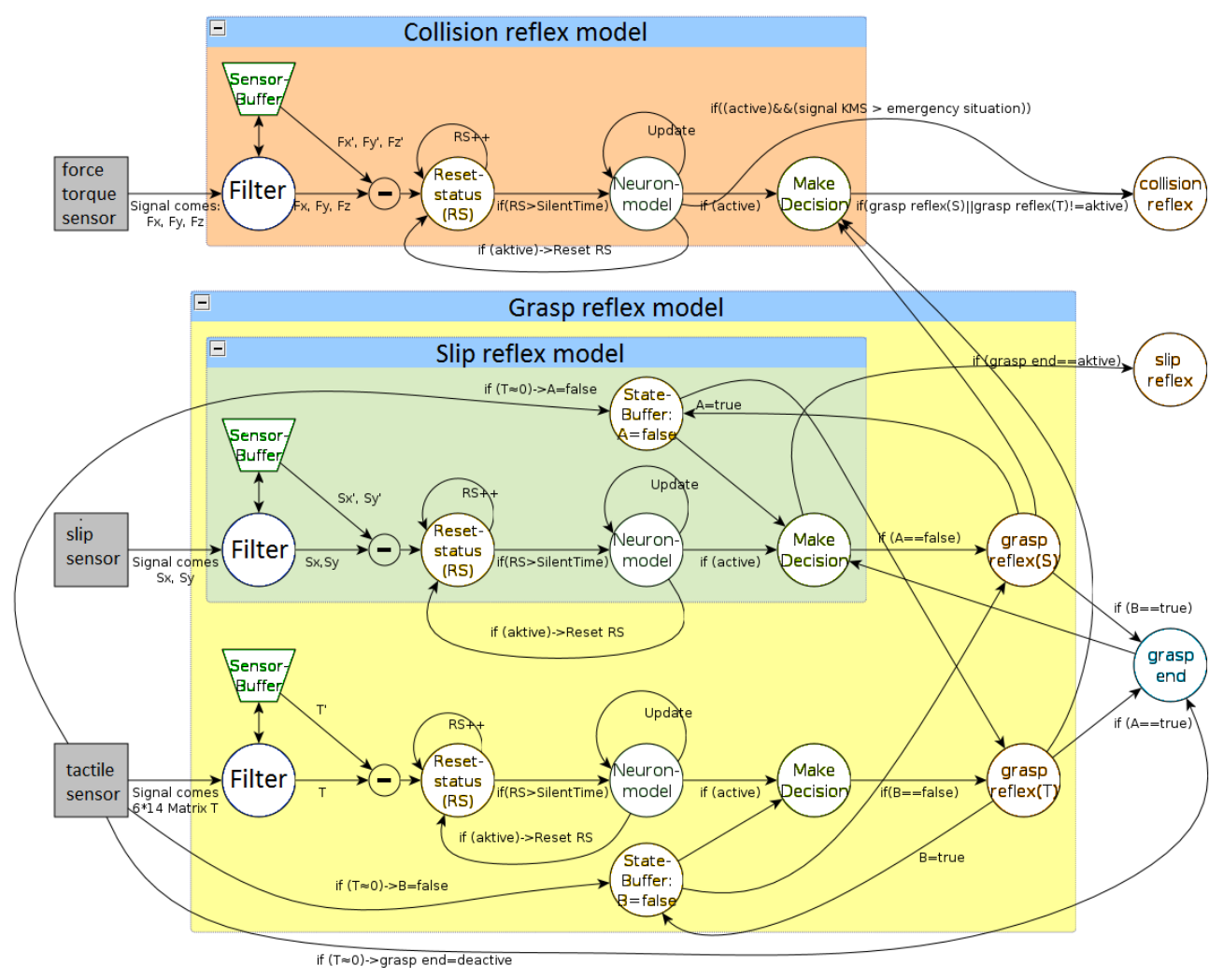

Fig. 2. Reflex coordination

state "grasp reflex (S)" or "grasp reflex (T)", respectively, change to "grasp end". As long as this state is active no other grasp reflex is allowed. The state buffer is reset as soon as the tactile sensor ceases sending signals. The development of the force-torque signals during the grasping process is stored to calculate the mass of the grasped object.

As soon as an activation signal is sent by a neuron, it is hyperpolarized for a short time and the refractory phase is imminent. That is why a counter is integrated into the neuron model, called "reset status". The counter starts at zero and accumulates over time as long as the condition "reset status $<$ silent time" is valid. During this time the neuron is locked and can not be activated.

\section{EXPERIMENTS}

For the experimental testing of the proposed reflex system, the robot platform of the Fraunhofer IOSB was used (Fig. 3). This demonstrator platform is built of two 7-Degrees-ofFreedom (DoF) AMTEC robot arms and one 2-DoF pan-tilt sensor head. The arms are anthropomorphic in their design and built of five rotation modules and one pan-tilt unit for the wrist. The modules are actuated by electronically commutated motors and harmonic drives. A two finger gripper and an anthropomorphic robot hand [21] are attached to the robot arms, each equipped which a force torque sensor that records the applying forces and torques in direction of $\mathrm{X}$-, $\mathrm{Y}$ - and Z-directions. The fingers of the two finger gripper are equipped with one tactile sensor array with $6 \times 14$ measuring textels and one optical slip sensor, which was developed

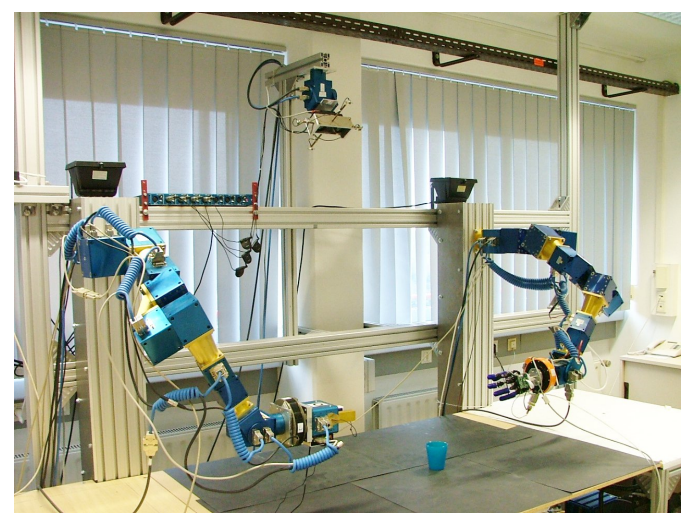

Fig. 3. Robot at IOSB

and patented at the IOSB [22]. This sensor is able to detect relative movements between the surface of the grasped object and the gripper. In principle it works like an optical computer mouse where a light source (LED) illuminates the objects surface so that its movement in X-and Y-direction can be detected. In addition to tactile, slipping and force-torque information, the position and the velocities of the robot arms are used as inputs for the developed reflex system.

\section{A. Determination of optimal threshold values}

The most important parameter of each neuron model is its threshold $v$ based on which the reflex is triggered. The lower the threshold, the easier the membrane potential $u(t)$ 
reaches the threshold and the reflex becomes more sensitive to input signals. To determine the thresholds of the neuron models tests have been performed using preliminary recorded data. At first, because of the fact, that no threshold is known, the activation of the model is secondary. The signals of the potentials have been simulated in this way and following results have been obtained:

The threshold of the grasp and slip reflex is determined by the measurement data. Regarding the fact, that these data is not as noisy as the one from the force-torque sensor, the thresholds can be set to lower values which increases the sensitivity of the reflexes. The thresholds used in the presented results are set to $\vartheta=2$, and the threshold for the two layer spiking neuron in the grasp reflex model are set empirically.

If the slip sensor detects a second slipping of the object, after the slip reflex has been active, this reflex is activated again immediately. For these tests, the silent time has been set to $t_{\text {silent }}=0.05 \mathrm{~s}$.

\section{B. Testing the grasp and slip reflex}

All three reflexes described above have been tested experimentally by placing objects of different nature (e.g. a wooden cylinder or a paper box) into the opened robot gripper. Grasping starts with either the tactile or the slip sensor triggering the grasp reflex. The slip reflex is activated after an object has been grasped successfully. In our testing scenario, no grasp planning was done, but only the mentioned reflexes have been used. Once an object is grasped, the signals of the slip sensor are used to detect unintentional movement of the grasped object. Two different possibilities exist which may result in this movement. The first is that the grip is too loose which results in a slipping of the object because of its mass, the other is that an external force other than gravitation is effecting the object. Those two situations have been simulated during the experiments by applying random forces to the grasped objects. If no object is grasped, the slip reflex is deactivated.

\section{EVALUATION}

Because of the fact that the slip reflex depends on a successful grasping operation, this reflex is tested in combination with the grasp reflex. All recorded data was evaluated, using the Matlab-Toolbox Gait-CAD [23]. For the slip reflex the changing of the sensor values has been under continuous observation. The silent time is set to a very low value because the slipping shall be detected at any time.

The grasp reflex uses the tactile sensor as well as the slip sensor. To realize this reflex the leaky integrate-and-fire model with dynamic threshold was used.

In this work we applied the single neuron model with relative input which works together with the slip reflex. In Fig. 4, a complete grasping operation is depicted. An activation signal is generated by the slip reflex at $2.5 \mathrm{~s}$ caused by placing an object into the gripper. Then the robot closes the gripper carefully until the first response by the tactile sensor is recorded at $9 \mathrm{~s}$. As long as the grasping is performed, no activation peak of the slip sensor is taken into account. This is changed after a successful termination of the grasping process. In response to a slip of the object of ca. $0.08 \mathrm{~mm}$ ( 1 count) the slip reflex is activated at $13 \mathrm{~s}$ and tightens the grasp preventing any further slipping of the grasped object. In Fig. 4 can be seen, that the activation signal $u_{f}$ occurs as soon as the gripper has contact to an object which starts the grasping process. At the end of the grasping, it can be seen, that, the higher the tactile signal, the more often fires the neuron. The adaption of the threshold suppresses unnecessary further firing of the neuron.

Negative in this scenario is, that the input depends on the area of contact in the gripper. This brings up some problems if very small objects should be grasped.

One possible effect, if the grasping was started by the slip reflex is, that the grasping stops with the first signal from the tactile sensor. At this moment, a secure grasp can not yet be guaranteed. That is why the grasping should not end at the first tactile sensor signal but depend on the frequency with which these sensor data arrives. Once the contact area does not increase any further and the threshold is adapted the neuron stops firing. This is when the grasping reflex is terminated.

Grasping also depends on the sensitivity of the material the tactile sensor is made of. In our case the stiffness was so high, that every object was already grasped tightly when the first sensor signal was recorded, but for further research and other sensors the above mentioned strategy may apply.

\section{CONCLUSIONS AND FUTURE WORK}

In this paper we presented a general framework for reflexes in humanoid robots. The design is based on leaky integrateand-fire neurons and can be specialized on any specific task. To test and evaluate this design in practice we chose three different reflexes known from humans and applied these to the general reflex module. In addition a reflex network was built that took the dependencies between different reflexes into account.

With these general reflex modules and its capability to form many kinds of different specialized reflexes we have a very flexible and general approach on applying reflexes to humanoid robots. This is part of our ongoing research and work is in progress to broaden the range of available reflexes and to show the significance of this approach against conventional techniques.

For the future we aim at combining these reflexes with neural structures called "Central-Pattern-Generators" (CPG) $[24,25]$ to synthesize movement trajectories and to trigger and adapt these by reflexes. CPGs have already been used successful for passive dynamic walkers [26,27]. Together with a Petri-net based system control this is part of a whole body control for humanoid robots.

\section{ACKNOWLEDGMENTS}

The work described in this paper was conducted within the collaborative research project "Humanoid Robots" (SFB588) funded by the German Research Foundation (DFG: Deutsche Forschungsgemeinschaft). 

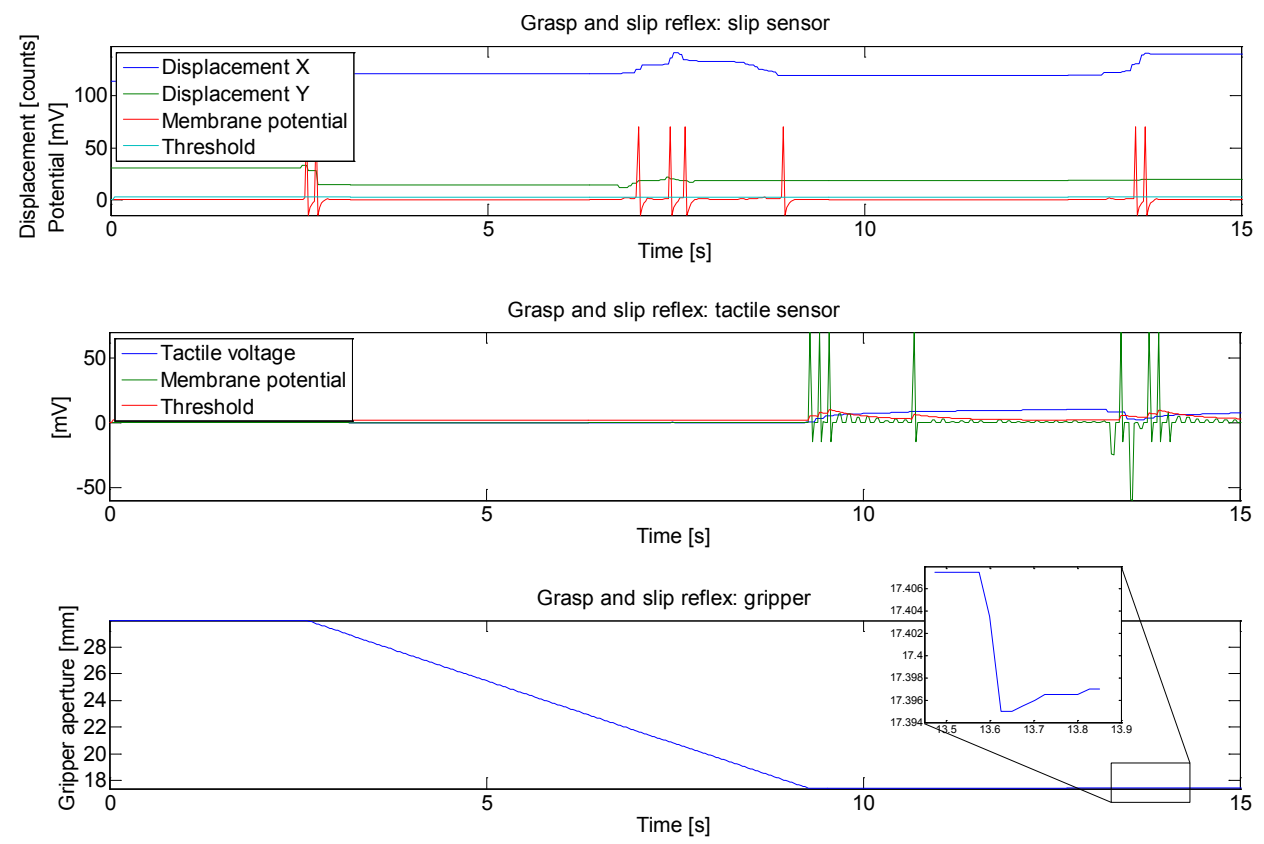

Fig. 4. Evaluation of slip and grip reflex

\section{REFERENCES}

[1] M. Folgheraiter and G. Gini, "Human-like Reflex Control for an Artificial Hand," BioSystems, vol. 76, pp. 65-74, 2004.

[2] S. Yigit, C. Burghart, and H. Wörn, "Applying reflexes to enhance safe human-robot-co-operation with a humanlike robot arm," Proc., 35th International Symposium on Robotics, 2004.

[3] H. Kawasaki, T. Mouri, J. Takai, and S. Ito, "Grasping of Unknown Object imitating Human Grasping Reflex," in Proc., 15th IFAC World Congress on Automatic Control. Elsevier Science, 2002.

[4] S. Shimoda and H. Kimura, "Biomimetic Approach to Tacit Learning based on Compound Control," IEEE Transactions on Systems, Man, and Cybernetics, Part B: Cybernetics, vol. 40(1), pp. 77-90, 2010.

[5] T. Asfour, K. Regenstein, P. Azad, J. Schröder, A. Bierbaum, N. Vahrenkamp, and R. Dillmann, "ARMAR-III: An Integrated Humanoid Platform for Sensory-Motor Control," in IEEE-RAS International Conference on Humanoid Robots (Humanoids 2006), 2006

[6] C. Burghart, R. Mikut, R. Stiefelhagen, T. Asfour, H. Holzapfel, P. Steinhaus, and R. Dillmann, "A Cognitive Architecture for a Humanoid Robot: A First Approach," in Proc., IEEE-RAS Conference on Humanoid Robots, Tsukuba, Japan, 2005, pp. 357-362.

[7] A. Lehmann, R. Mikut, and T. Asfour, "Petri Nets for Task Supervision in Humanoid Robots," in Proc., 37th International Symposium on Robotics (ISR 2006), München, 2006, pp. 71-73.

[8] G. Milighetti and H. Kuntze, "Fuzzy Based Decision Making for the Discrete-Continuous Control of Humanoid Robots," in Proc., IROS 2007, San Diego, California, 2007.

[9] L. F. Abbott and T. B. Kepler, "Model neurons: From HodgkinHuxley to hopfield," in Statistical Mechanics of Neural Networks, ser. Springer, 1990, vol. 368, pp. 5-18.

[10] A. Hodgkin and A. Huxley, "A Quantitative Description of Membrane Current and its Application to Conduction and Excitation in Nerve," The Journal of Physiology, vol. 117(4), pp. 500-544, 1952.

[11] R. FitzHugh, "Impulses and Physiological States in Theoretical Models of Nerve Membrane," Biophysical Journal, vol. 1 (6), pp. 445-466, 1961.

[12] J. Feng, "Is the Integrate-and-Fire Model good enough?-A Review," Neural Networks, vol. 14 (6-7), pp. 955 - 975, 2001.

[13] W. M. Kistler, W. Gerstner, and J. L. van Hemmen, "Reduction of the Hodgkin-Huxley Equations to a Single-Variable Threshold Model," Neural Computation, vol. 9 (5), pp. 1015-1045, 1997.

[14] W. Gerstner and W. Kistler, Spiking Neuron Models - Single Neurons, Populations, Plasticity. Cambridge University Press, 2002.
[15] J. Benda, L. Maler, and A. Longtin, "How to Model Spike-Frequency Adaptation in Integrate-and-Fire Neuron," in Bernstein Symposium, 2008

[16] M. J. Chacron, K. Pakdaman, and A. Longtin, "Interspike Interval Correlations, Memory, Adaptation, and Refractoriness in a Leaky Integrate-and-Fire Model with Threshold Fatigue," Neural Computation, vol. 15 (2), pp. 253-278, 2003.

[17] R. Azouz and C. M. Gray, "Dynamic Spike Threshold reveals a Mechanism for Synaptic Coincidence Detection in Cortical Neurons in vivo," in Proc., National Academy of Sciences of the United States of America, 2000, vol. 97 (14), pp. 8110-8115.

[18] R. Brette and W. Gerstner, "Adaptive Exponential Integrate-and-Fire Model as an Effective Description of Neuronal Activity," Journal Neurophysiology, vol. 94 (5), pp. 3637-3642, 2005.

[19] S. Wieser and K. Domanowsky, "Greifreflex und Stellmechanismus beim Säugling,” Journal of Neurology, vol. 175, pp. 520-527, 91957.

[20] W. Yan, "Nachbildung eines menschlichen Reflexbogens mittels künstlicher Neuronen Modelle," Master's thesis, Karlsruher Institut fr Technologie (KIT), 2009.

[21] S. Schulz, C. Pylatiuk, M. Reischl, J. Martin, R. Mikut, and G. Bretthauer, "A Lightweight Multifunctional Prosthetic Hand," Robotica, vol. 23(3), pp. 293-299, 2005.

[22] M. Bächlin, J. Beyerer, H.-B. Kuntze, and G. Milighetti, "Vorrichtung zum Schlupf-überwachten, kraftschlüssigen Ergreifen, Halten und Manipulieren eines Objektes mittels einer Greiferanordnung," German Patent, referenznummer: 102005032 502.5, 12.07.2005.

[23] R. Mikut, O. Burmeister, S. Braun, and M. Reischl, "The Open Source Matlab Toolbox Gait-CAD and its Application to Bioelectric Signal Processing," in Proc., DGBMT-Workshop Biosignalverarbeitung, Potsdam, 2008, pp. 109-111.

[24] Y. Chen, C. Bauer, O. Burmeister, R. Rupp, and R. Mikut, "First Steps to Future Applications of Spinal Neural Circuit Models in Neuroprostheses and Humanoid Robots," in Proc., 17. Workshop Computational Intelligence, 2007, pp. 186-199.

[25] C. Bauer, S. Braun, Y. Chen, W. Jakob, and R. Mikut, "Optimization of Artificial Central Pattern Generators with Evolutionary Algorithms," in Proc., 18. Workshop Computational Intelligence, 2008, pp. 40-54.

[26] P. Manoonpong, T. Geng, B. Porr, and F. Woergoetter, "The RunBot Architecture for Adaptive, Fast, Dynamic Walking," in Proc., IEEE International Symposium on Circuits and Systems (ISCAS), 2007, pp. 1181-1184.

[27] L. Righetti and A. J. Ijspeert, "Programmable Central Pattern Generators: An Application to Biped Locomotion Control," in Proc., IEEE International Conference on Robotics and Automation (ICRA), 2006, pp. 1585-1590. 